\title{
User Experience Research and Management of Online Advertising and Merchandising
}

\author{
Frank Y. Guo \\ 405 Howard St, San Francisco, CA 94105, USA \\ frank.guo@barclaysglobal.com
}

\begin{abstract}
Managing user experience of advertising on eCommerce sites poses unique challenges due to the need of balancing profiting and optimizing user experience. Merchandising on eCommerce sites is similar to online advertising, because users oftentimes do not perceive and interact with them differently due to their similar look and feel. This paper proposes a framework of user experience management, an approach towards user research, and a number of design recommendations for online advertising and merchandising.
\end{abstract}

Keywords: eCommerce, advertising, merchandising, eye tracking, user experience, usability.

\section{A Framework of User Experience Management of Online Advertising and Merchandising}

Having conducted extensive user research and provided user experience consulting around online advertising and merchandising when working at eBay Inc., I have found that managing user experience of advertising on eCommerce sites poses unique challenges. Defined broadly, user experience management in a corporate setting goes beyond just enhancing the user experience (e.g., usability and desirability of the product). It should also address how to leverage user experience to effect the ultimate business objective - profiting. In most cases these two objectives are in line with each other. For instance, improving the usability of software is likely to increase the sales of the software and bring in more profits. However, with regard to online advertising, conflicts arise between generating profits and improving user experience. The primary goal of having ads on an eCommerce site is to generate advertising revenue. Few would think having ads is an efficient way to improve user experience. Actually, because users typically visit eCommerce sites to buy items directly from those sites, their experience is likely to turn negative by seeing $3^{\text {rd }}$-party ads. Based on such dilemma, I will describe a framework of managing user experience around advertising, which can easily extend to merchandising.

\subsection{Balance between User Experience and Profits - The Big Picture}

Based on the above discussion, when managing user experience around online ads, we have to strike a balance between the business objective of increasing ads revenues 
and the user experience objective of having better usability, findability, and user satisfaction. That means we need to improve the noticeability and/or click through of ads, while introducing as little harm to user experience as possible. See Figure 1 for illustration. Ideally, well designed ads should have minimal negative impact on user experience, while attracting a decent amount of user attention, indicated by Ideal Ads in Figure 1. Too much attention to ads would distract shoppers from paying attention to the inventory of the site per se. Take one study I conducted as an example. Five ads designs were evaluated, illustrated by Ads version 1 through 5 in Figure 1. Based on the findings I qualitatively rated these five versions using this framework. Some of the ads were good at attracting user attention but caused negative user experience (e.g., version 3), and some were ineffective in attracting user attention while causing little negative user experience (e.g., version 4 and 5). In this hypothetical situation, among all these designs, Version 1 was the all-around best design, because it attracted a significant amount of user attention while avoiding too much negative experience. Version 2 and 3 fared worse than Version 1 because they led to more negative experience. Version 4 and 5 led to negligible negative experience, but unfortunately they attracted almost no attention, and thereby defeating the purpose of having ads on the web pages. We can further improve Version 1 by attracting a little less user attention (e.g., making the positioning less salient on the page) so that it does not take too much user attention away from the items that belong to the site itself and decreasing negative impact on user experience (e.g., making the ads sound more functional and less promotional). Using Figure 1 as an example, this means moving Ads version 1 towards the position of Ideal Ads. I will describe specific ways of improving ads design later in the paper.

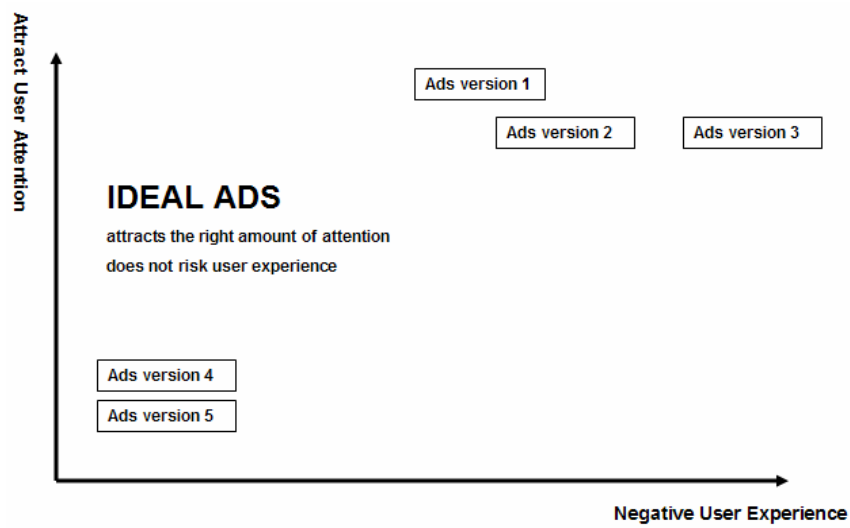

Fig. 1. A proposed model of evaluating advertising. Two factors, attracting user attention and avoiding negative user experience, should be considered together when evaluating user experience success around advertising.

Relative to advertising, there is less controversy around merchandising (e.g., featured items on eBay.com) on eCommerce sites. It is ok for merchandising to attract more user attention, because by promoting items on the same site to the shoppers, it is 
not "stealing" attention away from the site and undermining the core monetization model of the site, that is, to profit from selling its own merchandise.

The above framework needs revisions based on the context. For instance, it is ok to attract a lot of user attention when a search query does not return any results. In this case, having ads on the page is not competing with the inventory of the site as much as when there are many items found. It also depends on the monetization model of the ads and merchandising. If the monetization model is based on pay per click, then attracting user attention is not good enough for generating profits - click through is a better way of measuring ads effectiveness. Use case also matters. When a user comes to Amazon to perform research, he will be less likely to be annoyed by seeing ads than if he comes here to only shop for Amazon items.

\subsection{User Experience Management Objectives}

Consistent with the framework outlined above, based on my research and consulting experience, I developed a list of user experience management objectives that are uniquely suitable for online advertising and user research questions that we need to answer in order to help achieve these objectives:

- Increasing ads effectiveness. As user experience professionals, we should leverage our knowledge of user behavior to make ads more effective, by making users spontaneously notice and click the ads in their natural way of using the site. In terms of user research, we need to understand if users click the ads intentionally or by mistake, and whether they will re-visit the ads or avoid the ads after clicking them the first time.

- Avoiding confusion around ads. We should make sure that we do not introduce confusion to users by presenting ads on the site. In terms of user research, we need to understand whether users understand the nature of the ads, before and after clicking into the ads, and whether users mistake ads for merchandising given their apparent similarity.

- Avoiding negative attitude. We should decrease the amount of negative attitude towards ads, or even introduce positive experience towards ads if possible. In terms of research, we should assess user attitude throughout the entire process, including before and after they click into ads.

- Enhancing usefulness. We should design ads so that they are useful to the users. For instance, the ads might supplement the inventory when users cannot find what they are looking for within the inventory. In terms of research, we can probe users about use cases in which ads could be useful as well as observing if users spontaneously leverage ads when doing shopping.

- Avoiding interference with user tasks. Whereas there is little usability value in having ads per se, the presence of ads might hurt the usability of the rest of the site. In terms of research, we should investigate if ads interfere with users' completion of their intended tasks, such as looking at product reviews, checking out an item, etc.

- Preventing leaving the site. For pay per click ads, we would like users to click the ads. On the other hand, we would like users to return to the site after clicking into the ads. In terms of research, this requires us to observe user behavior after clicking into the ads. 
Most of the above objectives and research questions also apply to merchandising because of the apparent similarity between advertising and merchandising. On the other hand, we should be less concerned with avoiding negative attitude and preventing leaving the site when it comes to merchandising, because merchandising contains content or inventory that belongs to the site itself instead of a $3^{\text {rd }}$ party.

\section{User Research Approach around Online Advertising and Merchandising}

As mentioned above, in order to achieve the aforementioned user experience management objectives, we need to conduct research about how users interact with ads and merchandising first. User research around online advertising is rather different from typical user research such as inspecting software usability and evaluating the comprehensibility of web content, due to three reasons. First of all, there is no clear use case for ads per se - users do not come to an eCommerce site in order to see ads. Also, ads research generally does not focus on usability. Ads are not designed to accomplish particular tasks that users would typically perform, and therefore usability is not a main concern. So unlike when investigating software usability, we cannot ask users to perform a task that is intentional with regard to ads, such as "please find out the price of a camera that is NOT coming from an eBay seller", because this kind of task is not part of users' actual use cases on an eCommerce site. Another related point is that users probably do not spontaneously notice ads, so if we ask about their experience around ads, we will bias participant response by pointing their attention to something that they might not spontaneously notice. Given the unique nature of ads research, in this section I will introduce a recommended research approach and a set of common research questions. I will also highlight eye tracking as an optional research approach.

\subsection{Research Approach}

The above discussion points to the importance of studying spontaneous behavior in realistic contexts when conducting ads research. Whereas there are many research paradigms that could potentially accomplish the research goal, I would like to focus on in-lab user evaluation in this paper, because this approach provides the most indepth exploration into user behavior relative to non-interactive techniques such as surveys and quantitative remote user sessions. Below is a list of key techniques to employ when conducting in-lab ads research:

- Have participants do spontaneous shopping tasks. In order to understand real user behavior, we should use tasks that ask participants to do whatever that they would typically do on a site, such as "please do some online shopping at your own pace", without further specifications. Spontaneous shopping task allows participants to engage the eCommerce site in a natural manner and see realistic ads served by actual ads algorithms. In addition, relevance of ads might be an important factor in ads experience. If we force users to do a task that they do not care about, we lose the opportunity to see how relevance plays a role in their experience. Take shopping on eBay for an example, a participant might be a collector and would look for 
a rare antique clock on eBay. It is unlikely that any crafted task that the researcher can think of would match this participant's main use case when shopping on eBay, and will not get the participant engaged and exhibit natural shopping behavior.

- Use realistic testing environment. Unlike conventional user experience studies that heavily leverage low-fidelity prototypes, because we want to observe users' natural behavior, we should use a live site or high-fidelity testing environment, which allows users to search and browse as they wish and which will serve ads intelligently based on ads algorithms.

- Mask true intention during moderation. When moderating the sessions, please keep in mind that noticing ads is a low frequency behavior, and if we force participants to comment on ads or do things specifically related to ads, we will get findings that do not reflect their natural behavior. Therefore, when interviewing participants, it is important to reveal our intention of investigating about ads as late as possible. Even when probing about ads, we should avoid only focusing on the ads and let the participants talk about other things on the screen as well.

- Observe first, probe later. This technique is in line with the masking true intention point. As soon as we start probing, we start loosing the chance of understanding users' spontaneous behavior. Observing without asking questions is a great way to understand unbiased user behavior around ads. I would suggest not probe participants about the ads during the tasks, except when they mentioned the ads spontaneously. Only probe about ads after all tasks, to avoid biasing participants for later tasks.

- Avoid referencing to the ads as" ads" during moderation. This makes it possible to understand how participants naturally interpret the ads - they might not realize that these are ads at all.

- Have users perform similar shopping tasks multiple times. Because noticing ads is a low-frequency behavior and user behavior towards ads is dependent upon the context (e.g., what kind of information they are looking for on the site), asking users to do a variety of tasks will give us more chances of observing how users interact with ads in various contexts and when looking for various items to shop. For instance, we can ask users to shop for several items instead of just one item and observe how they behave around ads when shopping for them.

- Investigate how search results impact ads experience. For search-related tasks, user behavior around ads might be affected by the quantity and quality of the search results. For instance, when a user searches for an item on the site, if there is no item found, the user will be more likely to notice the ads than if there are many items found. The researcher should design a few tasks, some of which yield many search results and some of which yield few search results.

- Be cognizant of how context affects ads experience. Finally, the researcher needs to be cognizant of how context might affect user experience around ads, and design tasks and interpreting findings accordingly. For instance, users might be less tolerant about ads when doing transaction than when exploring shopping options. It is advisable to design tasks addressing different contexts separately and avoid making generalizations across contexts when interpreting research results. 


\subsection{Eye Tracking as an Optional Research Approach}

Visual attention is an important component to understanding user experience around ads and merchandising. For instance, is the user simply ignoring the ad or is paying attention to the ad without clicking on it? Such question can only be answered by a direct assessment of visual attention. Visual attention is rather spontaneous in nature, so oftentimes participants are not cognizant of where they looked at. This makes think aloud protocol less useful when investigating visual attention. Eye tracking, by tracing where the eyes fixate on the screen momentarily, is the most suitable research tool. I applied eye tracking to some advertising and merchandising studies that I conducted to complement the conventional evaluative techniques I used in those studies. Beware that eye tracking data alone are hard to interpret. For instance, users fixating at one area of the screen can either be interpreted as this area works well or this area confuses users - both possibilities lead to lots of visual attention. Therefore in order to help interpret eye tracking data and yield a comprehensive understanding of user behavior, eye tracking data should be cross referenced with think aloud protocol, interview, and observation of behavior. This does not mean that eye tracking session should be run at the same time when thinking aloud is elicited - talking while doing the tasks will affect eye movement and thus make the eye tracking data less valid. Typically, instead of running a stand alone eye tracking session, I use eye tracking to supplement the conventional user evaluation method that leverages think aloud protocol, probing, and observing of behavior in order to get additional information not collected by the conventional methods. To avoid bias and collect clean eye tracking data without the influence of moderation and learning, eye tracking session should precede conventional usability session. When running the eye tracking session, spontaneity is the key. Moderation, lack of interactivity in the prototype, think aloud protocol, and any kind of study-induced artificiality would make the eye tracking data less reflective of users' natural visual attention pattern.

There are various ways to interpret eye tracking information. Conventionally eye tracking data are analyzed quantitatively by aggregating data across a large sample of participants for each task [1]. The "heat map" that we saw from many eye tracking studies is an example of the quantitative data. The quantitative data can help us understand how much attention various screen regions attract user attention, but do not let us know about the process of how users discover information on the page. On the other hand, eye tracking data can be analyzed qualitatively one participant at a time on a task by task basis, much like how we analyze data for ethnographic research and one-to-one interviews. The qualitative data, when cross-referenced with think aloud protocol and observation of behavior, can provide rich insight into the process of information discovery. Both types of eye tracking data are rather valuable to advertising and merchandising research.

\section{Recommendations for Managing Online Advertising and Merchandising Experience}

Based on observation, interview, and eye tracking data collected around numerous websites from the many user evaluations I have conducted, I was able to derive consistent 
insights around advertising and merchandising experience. Many of these insights were also validated against findings from research conducted by others, which includes analytics, A/B tests, and market research studies conducted around same or similar websites about same or similar advertising and merchandising designs. I break down the insights in details below:

- Looking like ads leads to negative user experience and attracts less user attention. This might sound counterintuitive to many advertising professionals. After all, professional achievement by default should be measured by how crafted an ad design is. And the notion of "advertising campaign" is based on the idea that ads should be explicitly promoted to consumers in order to effect its marketing goals. However, at least in the eCommerce context, in order to attract user attention and avoid negative user experience, ads that look promotional (e.g., using polished graphs, containing generic motivational texts like "come and get one!") frequently annoyed the participants I interviewed when they did online shopping on the sites. By contrast, ads that were similar to the rest of the page attracted much more attention and produced much less negative experience. This is consistent with the well documented "banner blindness" phenomenon [2], according to which online users consciously or unconsciously ignore any thing that looks like a banner. In cognitive psychology, there is also such well-documented behavior called inattentional blindness, according to which people tend to ignore information that they are not actively attending to, even when the information is presented in a rather salient manner $[3,4]$. It is noteworthy that making ads look like the rest of the page might sound deceptive, but as long as the ads did feature actionable inventory, my participants exhibited negligible negative attitude, despite the fact that they realized they were misled into believing the ads were featured items on the site.

- Graphics might hurt, if too promotional or without pictures of the items. We often talk about graphic ads versus text ads, without realizing the distinction within graphic ads: graphic ads with pictures of specific items and graphic ads without pictures of specific items. Pictures of the specific items for sale attract user attention and do not give users the impression of being ads-like. By contrast, graphic ads without item pictures or with artistically rendered item pictures feel like too promotional and ads like, and attract less user attention and lead to negative user experience.

- Ads experience on eCommerce sites are different from that on search engines. Users are less annoyed by ads on search engines such as Google than on eCommerce sites, because the former is not a shopping destination. Users go to search engines in order to get to specific shopping sites. Advertising on search engines is consistent with this user goal. In contrast, eCommerce sites are shopping destinations - users visit these sites because the sites have unique values to their shopping and do not want to go elsewhere.

- Concrete items lead to better user experience and more user attention. Ads that feature concrete items with specific information such as price (e.g., "a 30G iPod at $\$ 200 ")$ and pictures of the item are more likely to attract user attention than ads that feature a generic store (e.g., "click here to check out our store, which has a large selection of iPods.") User attention always gravitates towards concrete and 
easy to understand information that forms the "don't make me think" experience, consistent with some previous research [5, 6].

- Habituation alleviates negative user experience. Multiple rounds of user evaluation of ads on the same sites showed that negative experience towards ads subdued as time passes. This finding is corroborated by direct comments made by participants, who explicitly mentioned that they were getting used to seeing ads on the sites. The overall internet experience also matters. Many participants mentioned that as more eCommerce sites feature ads, they began to feel less hostility towards seeing ads.

- Context-dependent advertising. Users are more annoyed by advertising on pages where they engage in targeted tasks (e.g., search results page, transaction page), and are more tolerant of ads on pages where users explore the content (e.g., homepage).

- Interfering with user tasks leads to negative user experience. Ads that interfere with user tasks (e.g., ads placed at top of search results) lead to worse user experience than ads that do not interfere with users tasks (e.g., ads placed at bottom of search results).

- Relevance helps improve user experience and attract user attention. Conventional wisdom tells us that relevance might be a key factor in the success of online advertising. My research corroborates this idea. In particular, both think aloud protocol and eye tracking revealed that ads relevant to users' tasks led to better user experience and attracted more user attention.

It is noteworthy that these insights are related to eCommerce sites that serve as a shopping destination such as eBay and Amazon, rather than comparison shopping sites such as shopping.com. Because users do not buy directly from comparative shopping sites, without conducting user research on them personally, I would hypothesize that they are more tolerant to $3^{\text {rd }}$-party ads on these sites.

Whereas these insights were derived primarily from my experience with eCommerce platform, they should also apply to other types of websites because they are related to some basic psychological mechanisms of online behavior. For instance, we can hypothesize that the reason that text ads work great on Google is because they look rather similar to the rest of Google content. This is consistent with the idea that ads should not stand out as ads on the page, and should blend well into the rest of the page.

\section{Discussion}

Proposals and recommendations presented in this paper are based on a basic premise - online advertising is more or less detrimental to user experience, and it is user experience professionals' job to balance profit making and user experience concerns. Some might argue that ads might actually improve user experience by providing more abundance to shopping experience. For instance, if a user cannot find an item on Amazon.com, $3^{\text {rd }}$-party ads can provide the user with alternatives. However, this is a risky way of enhancing shopping experience, as it undermines users' perception of the inventory quality of the site itself and might turn the user away from the site in the long run. Improving inventory and findability of the site itself is a much better approach than leveraging ads. So I would advise never to treat online ads as a means of improving user experience - it is always just a means to make profits. And if the 
business does not make significantly more money with online ads than without them, then just do not have them on the site. On the other hand, well designed merchandising is a safe way of enhancing users' shopping experience, as it features items from the site itself.

This paper offers many recommendations around improving online advertising. These recommendations are based on many rounds of user evaluations rather than an outcome of intuition, and are cross referenced with extensive quantitative research findings and analytics data. These recommendations are also the outcomes of crossreferencing between what participants said and what they did, rather than just listening to what they said. Some recommendations are rather counterintuitive. To summarize, the most important take-home message is that optimizing online advertising is not just about addressing the what, (i.e., what items the ads feature) - it has a lot to do with the how (i.e., the presentation and implementation of ads). Many ads professionals spend much time thinking about what message the ads should deliver. For instance, they might debate about whether to convey winning the bid or getting a cheap price when determining what messages an ad that features an auction item should deliver on eBay.com. But they tend not to pay an equal amount of attention to how the ads are substantively presented, such as visual treatment, page layout, the look and feel of the ads in relation to the rest of the page, length of the ads text, the quality of graphics, and so on, which also greatly impacts how well ads work. Another important piece of advice is that we should stop presenting ads like ads, that is, artsy and polished, and start making ads look like the rest of the eCommerce site, that is, showcasing concrete pictures and concrete item information and being functional, not flashy, in visual treatment. When making ads look similar to the rest of the page (e.g., featured items), so long as the ads feature concrete and actionable merchandise, users will not be upset by being misled, as indicated by my interviews with online shoppers. We also need to be clearly aware of the distinction between how users interact with ads online and how users interact with ads on other types of media, such as TV commercials - It is relatively much easier for users to neglect online ads. For instance, they do not even have to use the remote control to change the channel. Participants of my studies almost unconsciously filtered out ads from their attention. So in order to attract user attention to online ads, the ads experience should be designed in such way that makes users attend to ads without them even thinking about it. These recommendations also generally apply to merchandising, an area that is very similar to advertising in users' mind.

Disclaimer. The views expressed herein are those of the author and do not necessarily reflect those of Barclays Global Investors or its subsidiaries, management, or employees.

\section{References}

1. Jacob, R.J.K., Karn, K.S.: Eye Tracking in Human-Computer Interaction and Usability Research: Ready to Deliver the Promises. In: Radach, R., Hyona, J., Deubel, H. (eds.) The mind's eye: cognitive and applied aspects of eye movement research, pp. 573-605. North-Holland, Elsevier, Boston (2003) 
2. Norman, D.A.: Commentary: Banner Blindness, Human Cognition and Web Design, Internet Technical Group (1999)

3. Simons, D.J., Chabris, C.F.: Gorillas in our midst: Sustained inattentional blindness for dynamic events. Perception 28, 1059-1074 (1999)

4. Benway, J.P.: Banner blindness: The irony of attention grabbing on the World Wide Web. In: Proceedings of the Human Factors and Ergonomics Society 42nd Annual Meeting, vol. 1, pp. 463-467 (1998)

5. Krug, S.: Don't Make Me Think: A Common Sense Approach to Web Usability. New Riders Press, Indianapolis (2005)

6. Gladwell, M.: The Tipping Point: How Little Things Can Make a Big Difference. Back Bay Books, New York (2002) 